\title{
Empirical Analysis of Supervised and Unsupervised Filter based Feature Selection Methods for Breast Cancer Classification from Digital Mammograms
}

\author{
Subodh Srivastava \\ School of Biomedical Engg. \\ Varanasi-221005, UP, India
}

\author{
School of Biomedical Engg. \\ Neeraj Sharma \\ ndian Institute of Technology $(B H$
Varanasi-221005, UP, India
}

\author{
S.K. Singh \\ Department of CSE \\ Indian Institute of Technology(BHU) \\ Varanasi-221005, UP, India
}

\begin{abstract}
In the design and development of an automated CAD tool for breast cancer detection and diagnosis, the various steps include enhancement, segmentation, feature extraction, feature selection and classification. The feature selection plays an important role in the design of the said CAD tool as it aims towards the redundant feature elimination and relevant feature selection. The selected feature set also decides the efficacy of the chosen classifier for classification of mammograms. In literature, various filter based feature selection methods exists under unsupervised and supervised categories based on different basis criterion. The filter based feature selection methods ranks the extracted feature sets based on some criteria in descending order of their importance. The various methods produce different feature subsets which are associated with different performance measures. In this paper, an evaluation and comparative study of various unsupervised and supervised feature selection methods are presented for breast cancer classification from digital mammograms though various classifiers. The study aims towards finding out the better feature selection method and associated classifier which gives better performance.
\end{abstract}

\section{General Terms}

Digital Mammography, CAD Tool, Breast Cancer detection, Feature Selection, Pattern Classification

\section{Keywords}

Supervised feature selection methods, unsupervised feature selection methods, comparative study, classifier selection, CAD tool, breast cancer detection, MIAS database.

\section{INTRODUCTION}

Breast cancer is the most common form of cancer and it is the second leading cause of cancer death after lung cancer. Women in the India have about a 1 in 11 lifetime risk of developing invasive breast cancer. An early detection of breast cancer increases the survival rate and increases the treatment options. For early breast cancer detection, mammographic X-ray images are playing an important role due to its cost effectiveness and capability to detect the breast to cancer. Annual mammogram screenings, combined with other imaging based examination such as ultrasounds and MRIs, significantly increase the detection of cancer in women who had high risk of breast cancer. It has been reported [1] that out of more than 2,600 women who were at increased risk of breast cancer and they either had dense breast tissue and at least one other risk factor, such as a family history of the disease, 53 percent of the cancers were detected through mammograms[1-2]. Radiologists visually search mammograms for specific abnormalities. Screening mammography, radiographic imaging of the breast is currently the most effective tool for early detection of breast cancer. The various types of abnormalities which are indicator of breast cancer include micro-calcifications, tumours, and architectural distortions etc. Calcifications are deposits of calcium in breast tissue. Micro calcifications are usually associated with extra cell activity in the breast tissue and are grouped in clusters that can be a sign of developing malignant tumor. Scattered micro calcifications are usually a part of benign breast tissue. In mammograms calcifications are seen as bright dots of different sizes. The exact position of micro calcifications cannot be predicted, as well as their number [1] Breast tumors and masses usually appear in the form of dense regions in mammograms. Breast density is a measure of the extent of radio dense fibro glandular tissue in the breast which has the potential to be used as a predictor of breast cancer risk, it is a measure of how well tissue can be seen on mammogram [1]. Some tissue, such as the milk gland, is dense and appears white on a mammogram. This density makes it hard for medical professionals to observe tumors, which also appear white. Fatty tissue is less dense and appears clear on the mammogram, allowing better tumor detection [3]. Architectural distortion is defined as distortion of normal architecture with no definite mass visible, including speculation radiating from a point and focal retraction or distortion at the edge of the parenchyma. Architectural distortion of breast tissue can indicate malignant changes especially when integrated with visible lesion such as mass, asymmetry or calcifications. A mass is defined as a space occupying lesion seen in at least two different projections. If a potential mass is seen in only a single projection it should be called asymmetry or asymmetry density [2]. Masses have different density such as fat containing masses, low density , iso-dense, and high density; different margins such as circumscribed, micro lobular, obscured, indistinct; and speculated and different shapes such as round, oval, lobular, irregular round and oval [4-5]. Masses must be classified as benign or malignant. A typical benign mass has a round, smooth and well circumscribed boundary. A malignant tumor usually has a speculated, rough, and blurry boundary. There are subtle signs that can also lead to breast cancer diagnosis, such as architectural distortion and bilateral asymmetry [4-6]. Based on information discussed as above as well as other related pathological signs the radiologists arrive at conclusions to detect cancer from mammograms. In large scale mammogram screening program where numerous mammograms have to be examined by the various radiologists observers there may be chances of error in breast cancer detection, results may not be consistent due to human observer variability, and may be time consuming. Also in the 
examination of single mammogram there may be chances of error in breast cancer detection due to lack of expert professionals. Hence, an automated computer aided detection and diagnosis (CAD) [5-6] tool may be used for primary examination of mammograms for breast cancer detection and classification. If the CAD tool detects the cancer from mammogram the second opinion of radiologists may be sought for second opinion thus reducing the cancer detection and diagnosis time. In the design and development of a CAD tool for breast cancer detection the various primary steps involved include the restoration, enhancement, and segmentation of mammograms. The segmentation step aim towards the extraction of abnormalities such as microcalcifications, tumours, and architectural distortions. The next steps in the design of CAD tool include the features extraction from segmented image, feature selection method to select minimum redundant and maximal relevant features, and finally classification of mammograms in to normal and abnormal classes based on selected features. All these above mentioned steps play an important role in the design and development of a CAD tool.

In this paper, the feature selection and classification steps are addressed. This is due to the facts that in literature there are numerous feature selection and classification methods which can be used in the design of the said CAD tool and all these methods are associated with different performance measures due to their inherent characteristics and design features. Further, an empirical evaluation and comparative study of various supervised and unsupervised filter based features selection methods in terms of various classifiers are presented for mammogram dataset derived from MIAS database.

\section{FEATURE SELECTION: BACKGROUND}

In the design and development of a CAD tool for breast cancer detection from mammograms extracted feature sets from segmented mammogram are used for classification task. The extracted feature set may also contain redundant and nonrelevant features hence an appropriate feature selection method is applied. To select the relevant features from the large set of candidate features are called feature selection. If we extract $\mathrm{N}$ number of features for each mammogram in database then a feature vector is formed for each mammogram and all feature vectors for whole mammogram present in database constitute a feature matrix. After constituting a feature matrix, the next task is to select the most relevant features which best describes the mammographic image content from the overall feature space. This can be done by computing some sort of feature scores for each images and sorting or ranking them according to their relevance. The main objective of features selection are that it reduces the dimensionality of feature space, speed up and reduce the cost of learning algorithms, improve the predictive accuracy of classification algorithm, and also improve the visualization and the comprehensibility of the induced concepts. The feature selection algorithms may be based on three major criterions such as based on some evaluation measure; based on search organization; and based on the generation of successors [7, 9] as presented in Table 1. The features selection algorithms [9-10] are broadly divided into three categories: filter based, wrapper based, and hybrid.

\section{Table 1. Feature selection methods characterizations based on different criterion and their types}

\begin{tabular}{|l|l|}
\hline Characterization & Types \\
\hline
\end{tabular}

\begin{tabular}{|c|c|}
\hline criterion & \\
\hline \multirow[t]{5}{*}{ Evaluation measure } & Distance based \\
\hline & Divergence based \\
\hline & Information theoretic based \\
\hline & Dependence measure based \\
\hline & Accuracy based \\
\hline \multirow[t]{3}{*}{ Search organization } & Exponential \\
\hline & Sequential \\
\hline & Random \\
\hline \multirow{5}{*}{$\begin{array}{l}\text { Generation of } \\
\text { successors }\end{array}$} & Forward selection \\
\hline & Backward selection \\
\hline & Compound selection \\
\hline & Random selection \\
\hline & Weights based selection \\
\hline
\end{tabular}

Filter based features selection method use general characteristics of the data independently from the classifier for the evaluation process. In wrapper based methods, the evaluation process is classifier-dependent and uses the learning algorithm as a subroutine. The general argument in favour of this scheme is to equal the bias of both the feature selection algorithm and the learning algorithm that is used later to assess the goodness of the solution. The main disadvantage associated with wrapper based scheme is the extra computational cost that comes from calling classifier algorithm to evaluate each subset of considered features. For optimal feature selection in wrapper based method, the classifier error rate is minimized and a feature subset associated with minimum misclassification error is selected. The wrapper based feature selection method losses its generality, but gain accuracy towards the classification task and is computationally extensive. The hybrid models use both filtering and wrapping methods for improving the performance of the selection process. Evaluating the discrimination power of the individual feature is a key operation in feature selection processes. Several methods may be used to evaluate the discrimination power of a feature which includes distance, divergence, information, dependence, and accuracy based criterions. In information theoretic based approaches the mutual information is used for measuring the feature and data relations. Inter cluster and inner-cluster affinity characterizes the relationship between features and classes; thus, they are for different attributes of feature-data relations.

All these three categories (filter, wrapper, and hybrid) [9] can be divided into supervised and unsupervised basis; further supervised and unsupervised can be categories as multivariate and univariate. In supervised learning, the data is assigned to be known before computation and are used in order to 'learn' the parameters that are really significant for those clusters. Here each object in the data set comes with a pre assigned class label. The main task is to train a classifier to do the labelling but often the labelling process cannot be described in an algorithmic form, hence the machines is equipped with 
learning skills and present the labelled data to it. The classification knowledge learned by the machine in this process might be obscure, but the recognition accuracy of the classifier will be the judge of its adequacy. In unsupervised learning, the datasets are assigned to segments, without the clusters being known. In literature, numerous clustering algorithms have been and are being developed for unsupervised learning. Some examples include k-means clustering and fuzzy c-means clustering approaches [8]. In unsupervised learning approach, different algorithms exists with different structures for the same set of data and the disadvantages and advantages of these approaches are that there is no ground truth against which the results of an algorithm can be compared. The only indication of how good the result is probably the subjective estimate of the user. Further the feature selection process may be defined for univariate and multivariate data as the case may be for both types of learning approaches viz. supervised and unsupervised. In univariate data analysis, it is assumed that the response variable is influenced only by one other factor whereas in multivariate data analysis it is assumed that the response variable is influenced by multiple factors and even combinations of factors. In mathematics, univariate refers to an expression, equation, function or polynomial of only one variable. Objects of any of these types but involving more than one variable may be called multivariate.

\subsection{Filter based feature selection methods}

In this paper, an empirical evaluation of filter based feature selection methods [9-10] are presented in terms of various classifier performance measures. As discussed above, the filter based feature selection methods may be broadly categorized into two categories namely unsupervised and supervised defined for univariate or multivariate data. A classification of some of the popular filter based feature selection methods under these categories are presented in Table 2. Further classification of filter based feature selection methods can be done according to the basis criterion used for evaluating the discrimination power of features. The various types of evaluation functions are presented in Table 3. The categorization of some of the popular filter based feature selection methods which uses different basis criterion [8-9, 11--17] or evaluation function are presented in Table 4.

Table 2. Classification of some filter based feature selection methods on the basis of supervised and unsupervised learning approaches

\begin{tabular}{|l|c|c|c|l|}
\hline \multirow{2}{*}{$\begin{array}{l}\text { Filter based feature } \\
\text { Selection methods }\end{array}$} & \multicolumn{2}{|c|}{ Supervised } & \multicolumn{2}{c|}{ Unsupervised } \\
\cline { 2 - 5 } Relief F [11] & Multivariate & Univariate & Multivariate & Univariate \\
\hline mRmR [12] & Yes & No & No & No \\
\hline FCBF [13] & Yes & No & No & No \\
\hline GFLIP [14] & Yes & No & No & No \\
\hline Fisher score [8] & No & Yes & No & No \\
\hline SVM- RFE [15] & Yes & No & No & Yes \\
\hline t-test [8-9] & No & No & Yes \\
\hline $\begin{array}{l}\text { Bhattacharya distance } \\
\text { [8-9] }\end{array}$ & No & No & Yes \\
\hline $\begin{array}{l}\text { Wilcoxon paired test } \\
\text { 8-9] }\end{array}$ & No & No & No & Yes \\
\hline ROC based [8-9] & No & No & No & Yes \\
\hline Entropy based [8-9] & No & No & Yes & No \\
\hline PCA [8] & No & No & No \\
\hline Laplacian Score [16] & No & No & & Yes \\
\hline
\end{tabular}

Table 3. Evaluation functions used by filter based feature selection methods [9]

\begin{tabular}{|l|l|}
\hline Basis Criterions/ Evaluation function used & Examples \\
\hline Distance based measures & Euclidean distance \\
\hline Information theory based measures & Entropy, information gain, mutual information \\
\hline Data dependency measures & Correlation coefficient \\
\hline Consistency based measures & Minimum features bias \\
\hline
\end{tabular}

Table 4. Brief description of some of the popular filter based feature selection methods 


\begin{tabular}{|c|c|c|}
\hline \multirow[t]{6}{*}{$\begin{array}{l}\text { Supervised feature selection } \\
\text { methods }\end{array}$} & Fisher Score [8] & $\begin{array}{l}\text { Distance based, univariate filter method } \\
\text { evaluating each feature individually }\end{array}$ \\
\hline & ReliefF [11] & $\begin{array}{l}\text { A multivariate filter method taking into } \\
\text { account dependencies between features. }\end{array}$ \\
\hline & $\mathrm{mRmR}[12]$ & $\begin{array}{l}\text { Information theory based and uses mutual } \\
\text { information (MI) }\end{array}$ \\
\hline & FCBF [13] & $\begin{array}{l}\text { Based on information gain, FCBF is Fast } \\
\text { Correlation-Based Filter }\end{array}$ \\
\hline & SVM-RFE [15] & $\begin{array}{l}\text { SVM-RFE method ranks features based } \\
\text { on their corresponding coefficients in the } \\
\text { SVM classifier }\end{array}$ \\
\hline & GFLIP (greedy feature flip) [14] & $\begin{array}{l}\text { Margin based greedy feature selection } \\
\text { algorithm }\end{array}$ \\
\hline \multirow{7}{*}{$\begin{array}{l}\text { Unsupervised rank based } \\
\text { feature selection methods }\end{array}$} & ttest scores & \multirow{5}{*}{$\begin{array}{l}\text { Statistical, rank based feature selection } \\
\text { methods [8-9] }\end{array}$} \\
\hline & Bhattacharyya distance & \\
\hline & Wilcoxon signed-rank test & \\
\hline & Entropy Rank feature & \\
\hline & Receiver operating characteristic (ROC) based & \\
\hline & Principal component analysis (PCA) [8] & $\begin{array}{l}\text { PCA finds a linear projection of high } \\
\text { dimensional data into a lower dimensional } \\
\text { subspace such as: the variance retained is } \\
\text { maximized; the least square reconstruction } \\
\text { error is minimized. }\end{array}$ \\
\hline & Laplacian Score based [16] & $\begin{array}{l}\text { Laplacian Score (LS) is an unsupervised } \\
\text { feature selection algorithm based on } \\
\text { Laplacian Eigen maps and Locality } \\
\text { Preserving Projection. The basic idea of } \\
\text { LS is to evaluate the features according to } \\
\text { their locality preserving power. }\end{array}$ \\
\hline
\end{tabular}

\section{METHODS AND MODELS}

This paper presents the empirical analysis of supervised and unsupervised filter based feature selection methods for breast cancer classification from digital mammograms. For experimentation purposes the 322 mammogram images available in MIAS database were used. For the evaluation of the feature selection and classification methods following steps were used in the design of the CAD tool.

3.1 Enhancement of mammograms: The first step in the design of the CAD tool is enhancement of mammograms for highlighting the abnormalities such as micro-calcifications, tumours etc. In this step, a contrast limited histogram equalization (CLAHE) [18] method combined with unsharp masking and crispening [19] were used.

3.2 Segmentation of mammograms: For the segmentation of abnormalities present in the mammograms a modified fuzzy c-means based thresholding [20] method was used.
3.3 Feature Extraction [21]: After segmentation process, the 88 hybrid features were extracted for each of the segmented mammogram. The various features extracted belong to the various categories which are histogram based features, shape based features, texture features, wavelet based features, and Gabor features. The brief descriptions of extracted hybrid features (total 88 numbers) from each segmented mammograms are given as below:

Histogram based features [19,21] (F1-F16]: Mean, Standard Deviation, Gray Level Local Variance, Variance, Kurtosis, Skewness, Entropy, Histogram Range, Mean Absolute Deviation (MAD), Second Order Moment (Var), Mean of Zscore, Normalized gray level variance, Mean energy of gradient, Threshold Gradient, Squared gradient, Spatial Frequency.

Texture Features [21-22]: (F17-F37) Angular Second Moment (ASM), Contrast, Correlation, Variance, Standard deviation, Dissimilarity, IDM, Energy, Entropy, Cluster shade, Cluster Prominence, Sum Average, Sum Entropy, Sum Variance, Difference Variance, Difference Entropy, Information measure and others. These features have been 
derived from the gray level co-occurrence matrix (GLCM) probabilities.

Geometric or shape Features [19, 21]: (F38-F44) Area, Perimeter, Orientation, Equivalent diameter, Euler number, Eccentricity, Image Curvature. Geometric features describe the geometric properties of the region of interest (ROI). It is represented as a collection of pixels in an image.

Wavelet based features [21, 23-24]: (F45-F52) Mean Entropy, Energy, Contrast, Homogeneity, Sum of wavelet coefficients, Variance of wavelet coefficients, Wavelet ratio.

Gabor Features [21, 25]: (F53-F88) Mean square energy Orientation i.e. Mean Amplitude (at $3^{\text {rd }}$ level of decomposition having 6 orientations i.e. 36 features)

3.4 Feature Selection: The next step in the design of the CAD tool is the feature selection and evaluation of various filter based supervised and unsupervised feature selection methods are the main focus of this paper. A brief description of various feature selection methods are presented in section 2. The various supervised filter based feature selection methods considered in this paper are as follows: ReliefF [11], mRmR [12], FCBF [13], GFLIP [14], Fisher score [8], and support vector machine -recursive feature elimination (SVM-RFE) [15]. Further, the various unsupervised filter based feature selection methods in consideration includes various rank based feature selection methods [8-9] such as t-test scores, Bhattacharyya distance, Wilcoxon signed-rank test, entropy based, receiver operating characteristic (ROC) based; principal component analysis (PCA) based [8], and Laplacian score [16] based methods.

3.5 Classification [8]: The various supervised classifiers used for the evaluation of various feature selection methods for the design and analysis of the CAD tool include Naïve Bayes , k-nearest neighbour (k-NN), linear discriminant analysis (LDA), artificial neural networks (ANN), and support vector machines [26] for its various kernel functions such as linear, RBF, quadratic, polynomial, and multilayer perceptron (MLP).

\section{RESULTS AND ANALYSIS \\ 4.1 Dataset description}

For evaluating the performance measures of various filter based feature selection methods, the mammograms available in MIAS database were used. The MIAS database [27] consists of 322 mammographic images which contain 207 images for normal cases and 115 images for abnormal cases .The MIAS database images includes radiologist's "truth"markings on the locations of any abnormalities that may be present. Normal cases mean only normal breast tissues. Abnormal cases include both benign and malignant images, which includes radiologist's "truth" markings on the locations of any abnormalities that may be present. The various pathological features present in abnormal cases are as follows [27]:

Character of background tissue: Fatty, Fatty-glandular, and Dense-glandular.

Class of abnormality present: Calcification, Welldefined/circumscribed masses, speculated masses, miscellaneous other ill-defined masses, architectural distortion, asymmetry.

\subsection{Performance measures}

The performance measures used for the evaluation of the feature selection methods in terms of chosen classifier include accuracy, sensitivity, specificity, balanced classification ratio (BCR), F-measure, and area under curve (ROC). These measures are defined in terms of confusion matrix elements TP(true positive), TN (true negative), FP (false positive), and FN (false negative) as follows:

$$
\begin{aligned}
\text { Accuracy } & =\frac{T P+T N}{T P+T N+F P+F N} \times 100 \\
\text { Sensitivity } & =\frac{T P}{T P+F N} \\
\text { Specificity } & =\frac{T N}{F P+T N}
\end{aligned}
$$

Balanced classification rate,

$$
B C R=\frac{1}{2}[\text { Sensitivity }+ \text { Specificity }]
$$

F-measure is harmonic mean between precision and recall and defined as

$$
F_{-} \text {measure }=2 *\left[\frac{(\operatorname{Pr} \text { ecision } \times \operatorname{Re} \text { call })}{(\operatorname{Pr} \text { ecision }+\operatorname{Re} \text { call })}\right]
$$

where Precision $=\frac{T P}{T P+F P}$ and Recall $=$ Sensitivity .

Area under curve (AUC) is defined as the area under the receiver operating characteristic (ROC) curve.

\subsection{Performance analysis and discussions}

For testing purposes, top 50 most relevant features out of total 88 hybrid features were selected for each feature selection method. For hybrid features, the feature matrix size reduced to $322 \times 50$ after feature selection. The images in MIAS database were categorized into two groups namely normal and abnormal images and a class or group of size $322 \times 1$ was used. In next step, 10 -fold cross-validations were applied to split the $322 \times 50$ size feature matrix and $322 \times 1$ class size into two sets viz. train and test data sets and groups respectively. The size of train feature matrix was $289 \times 50$ and that of the test feature matrix was $33 \times 50$. Finally, selected feature subsets by each of the feature selection methods were used to evaluate the performances of the various supervised classifiers to find the best combination of feature selection method and classification model for the said CAD tool. For training, testing, and measuring the classifier's performance measures, 10 -fold cross-validations were used and the results are reported for the average of 100 runs for all the cases presented in this paper.

Tables 5, present the comparative analysis of the various supervised feature selection methods in consideration. Fig 1 shows comparison of supervised filter based feature selection methods in terms of classifier's maximum accuracy, sensitivity, and specificity values, and Fig 2 shows comparison of supervised filter based feature selection methods in terms of classifier's maximum AUC, BCR, and Fmeasures values. From Table 5 and Figs. 1-2 following observations are made:

- ReliefF feature selection method is performing better for SVM-MLP classifier with accuracy $84.37 \%$;

- $\quad \mathrm{mRmR}$ feature selection method is performing better for SVM-MLP classifier with accuracy $87.50 \%$; 
- FCBF is performing better for SVM-Quadratic with $78.12 \%$, however SVM-RBF is associated with $87.50 \%$ of accuracy but sensitivity value is 1 and specificity value is 0 giving boundary conditions i.e. SVM-RBF is not capable of classifying both positive and negative samples simultaneously for the dataset in consideration.

- GFLIP feature selection method is performing better for k-NN classifier with accuracy $81.25 \%$;

- $\quad$ Fisher score based method is performing better for SVMMLP classifier with $81.81 \%$ of accuracy,

- $\quad$ SVM-RFE is performing better for k-NN classifier with $84.37 \%$ of accuracy.

- The better performance of most of the supervised filter based classifier are associated with either SVM-MLP classifier or k-nearest neighbour classifier $(\mathrm{k}-\mathrm{NN})$ for $\mathrm{k}=5$. Therefore, from above observations it can be concluded that $\mathrm{mRmR}$ based feature selection method is performing better with $87.50 \%$ accuracy for SVM-MLP classifier followed by SVM-RFE and ReliefF methods with $84.37 \%$ accuracy of each for SVM-MLP and k-NN classifiers respectively. On further observation of other values of performance measures, in addition to accuracy of classifier, following conclusions are made:-

- Performance measures for mRmR for SVM-MLP classifier are:

Accuracy: $87.50 \%$, Sensitivity: $85 \%$, Specificity: $90 \%$, AUC: 0.76, BCR: 0.88, and F-measure: 0.90 .

- Performance measures for SVM-RFE for k-NN classifier are:

Accuracy: $84.37 \%$, Sensitivity: $83.33 \%$, Specificity: 87.50\%, AUC: 0.6111, BCR: 0.8541, and F-measure: 0.8888

- $\quad$ Performance measures for ReliefF for SVM-MLP classifier are:

Accuracy: $84.37 \%$, Sensitivity: $84.37 \%$, Specificity: $60 \%$, AUC: 0.71, BCR: 0.77, and F-measure: 0.89 .

In view of above observations the order of supervised filter based feature selection methods in terms of their performance are as follows:

$\mathrm{mRmR}>\mathrm{SVM}-\mathrm{RFE}>$ ReliefF $>$ Fisher score $>$ GFLIP $>$ FCBF .

Therefore, from above observations it can be concluded that $\mathrm{mRmR}$ feature selection is performing better in comparison to all other methods closely followed by SVMRFE and ReliefF methods.

Further, Table 6 presents the performance comparison of various unsupervised filter based feature selection methods in consideration. Fig 3 shows comparison of unsupervised filter based feature selection methods in terms of classifier's maximum accuracy, sensitivity, and specificity values, and Fig 4 shows comparison of unsupervised filter based feature selection methods in terms of classifier's maximum AUC, $\mathrm{BCR}$, and F-measures values.

From Table 6 and Figs 3-4 following observations are made:

- $\quad \mathrm{t}$-test based feature selection method is performing better for ANN classifier with accuracy $87.50 \%$ followed by SVM-Polynomial classifier with accuracy $81.81 \%$;

- Bhattacharya rank feature based method is performing better for k-NN classifier with accuracy $81.81 \%$;

- Wilcoxon rank feature based method is performing better for k-NN classifier with $84.37 \%$ accuracy;

- $\quad$ ROC rank feature is performing better for SVM-MLP classifier with accuracy $84.375 \%$;

- $\quad$ Entropy rank feature based method is performing better for k-NN classifier with accuracy $75 \%$;
- PCA based feature selection method is performing better for $\mathrm{k}-\mathrm{NN}$ classifier with accuracy $81.81 \%$;

- Laplacian Score based method is performing better for kNN classifier with $81.25 \%$ accuracy.

- Most of the unsupervised feature selection methods are performing better for k-NN classifier. Some of them are performing better for ANN and SVM-MLP classifier.

Hence, from Table 6, it can be concluded that t-test method is associated with better performance of $87.50 \%$ for ANN classifier followed by Wilcoxon rank feature based method for k-NN classifier with $84.37 \%$ accuracy.

\section{CONCLUSIONS}

In this paper, an empirical analysis of various supervised and unsupervised filter based feature selection methods for various classifiers was presented for breast cancer classification from mammograms. For experimentation purposes 322 mammograms available in MIAS database were used. The total of 88 hybrid features were extracted from each mammogram in database after applying enhancement and modified fuzzy c-means based segmentation approach. The efficacy of various filter selection method were evaluated for top 50 features out of 88 hybrid features extracted from 322 mammograms in terms of accuracy, sensitivity, specificity, BCR, F-measure and AUC. From the obtained results, it can be concluded that $\mathrm{mRmR}$ based feature selection method is performing better with $87.50 \%$ accuracy for SVM-MLP classifier in comparison to other methods followed by SVMRFE and ReliefF methods under supervised category. Under unsupervised category, t-test method is performing better with $87.50 \%$ accuracy for ANN classifier followed by Wilcoxon rank feature based method for k-NN classifier with $84.37 \%$ accuracy. In addition to evaluation of feature selection methods, it is also observed that in most of the cases k-NN and SVM-MLP classifiers are performing better. The overall performance of $\mathrm{mRmR}$ feature selection method is better in comparison to all filter based feature selection methods in consideration under both supervised and unsupervised categories.

\section{REFERENCES}

[1] Cancer facts and figures 2011. http://www.cancer.org/Cancer/BreastCancer /DetailedGuide/ breast-cancer-key-statistics.

[2] American College of Radiology (ACR) (2003): ACR Breast Imaging Reporting and Data System, Breast Imaging Atlas, 4th Edn., Reston, VA, USA.

[3] Subashini T.S. , Ramalingam V., Palanivel S. 2010. Automated assessment of breast tissue density in digital mammograms. Computer Vision and Image Understanding. 114, 33-43.

[4] Rangayyan, R.M., Ayres, F.J., Desautels, J.E.L 2007. A Review of Computer-Aided Diagnosis of Breast Cancer: Toward the Detection of Subtle Signs. Journal of the Franklin Institute. 312-348.

[5] Bozek J., Mustra M., Delac K., and Grgic M. 2009. A Survey of Image Processing Algorithms in Digital Mammography. Rec. Advances in Mult. Sig. Process. And Commn. (Grgic M. Eds.) SCI 231. Springer-Verlag Berlin Heidelberg. 631-656.

[6] Tang J., Rangayyan R., Xu J., Naqa I. El, Yang Y. 2009. Computer-aided detection and diagnosis of breast cancer with mammography. IEEE Transactions Recent 
advances, Information Technology in Biomedicine, 13(2), 236-251.

[7] Srivastava S., Sharma N., Singh S.K. 2013. Image Analysis and Understanding Techniques for Breast Cancer Detection from Digital Mammograms. In Research developments in image processing and computer vision, Srivastava R. Singh S.K., and Shukla K.K. (Eds.), Chapter 8, pp. 123-148. IGI Global, USA.

[8] Duda R. O., Hart P.E. and Stork D.G. 2000. Pattern Classification (2nd Edition). Wiley, India.

[9] Guyon I. , and Elisseeff A. 2003. An introduction to variable and feature selection. Journal of Machine Learning Research, 3:1157-1182.

[10] Kohavi R. and John G.H. 1997. Wrapper for feature subset selection. Artificial Intelligence. 97(1-2), 273324..

[11] Marko R. S., Igor K. 2003. Theoretical and empirical analysis of Relief and ReliefF. Machine Learning Journal. 53, 23-69.

[12] Peng H., Long F., and Ding C. 2005. Feature selection based on mutual information: criteria of maxdependency, max- relevance, and min-redundancy. IEEE Transactions on Pattern Analysis and Machine Intelligence. 27( 8), 1226-1238.

[13] Yu L. , and Liu H. 2003. Feature selection for highdimensional data: A fast correlation-based filter solution. In proceedings of the ICML'03 conference. 856-863.

[14] Gilad-Bachrach R., Navot A., and Tishby N. 2004. Margin based feature selection - theory and algorithms. In Proceedings of $21^{\text {st }}$ ICML conference. 337-344.

[15] Furey T.S., Cristianini N., Duffy N., Bednarski D.W., Schummer M., and Haussler D. 2000. Support Vector Machine Classification and Validation of Cancer Tissue Samples Using Microarray Expression Data. Bioinformatics. 16(10), 906-914.

[16] He, X., Cai, D., Niyogi, P. 2005. Laplacian score for feature selection. In: Advances in Neural Information Processing System, vol. 17. MIT press, Cambridge.
[17] Yang F. and Mao K.Z. 2011. Robust Feature Selection for Microarray Data Based on Multicriterion Fusion. IEEE/ACM Transact. On Comput. Biology and Bioinformatics. 8( 4), 1080-1092.

[18] Zuiderveld, K. (1994) 'Contrast Limited Adaptive Histogram Equalization', Graphic Gems IV. San Diego: Academic Press Professional, pp. 474-485.

[19] Jain, A.K. 2006. Fundamentals of Digital Image Processing. PHI, India.

[20] Cai, W., Chen, S. and Zhang, D. 2007. Fast and robust fuzzy c-means clustering algorithm incorporating local information for image segmentation. Pattern Recognition. 40( 3), 825-838.

[21] Srivastava, S., Sharma, N., Singh, S.K. and Srivastava, R. 2013. Design, analysis and classifier evaluation for a CAD tool for breast cancer detection from digital mammograms. Int. J. Biomedical Engineering and Technology. 13( 3), 270-300.

[22] Haralick, R.M., Shanmugam, K. and Dinstein, I. 1973. Textural features for image classification. IEEE Transactions on Systems, Man, and Cybernetics. SMC-3 (6), 610-621.

[23] Gonzalez, R.C. 2003. Digital Image Processing. Prentice Hall, India.

[24] Huang, K. and Aviyente, S. 2008. Wavelet Feature Selection for Image Classification. IEEE Transactions on Image Processing, 17( 9),1709-1720.

[25] Grigorescu, S.E., Petkov, N. and Kruizinga, P. 2002. Comparison of Texture Features Based on Gabor Filters. IEEE Transactions on Image Processing. 11(10),11601167.

[26] V. Vapnik 1998. Statistical Learning Theory. John Wiley \& Sons, USA.

[27] Mammographic Image Analysis Society (MIAS) database. 2013.http://www.mammoimage.org/databases/

Table 5. Comparison of supervised filter based feature selection methods in terms of various classifier performance measures for the given mammogram data set

\begin{tabular}{|c|c|c|c|c|c|c|c|c|}
\hline \multirow{2}{*}{$\begin{array}{l}\text { Feature } \\
\text { Selection } \\
\text { Methods }\end{array}$} & \multirow{2}{*}{\multicolumn{2}{|c|}{$\begin{array}{l}\text { Classifier for } \\
\text { detection }\end{array}$}} & \multicolumn{6}{|c|}{ Classifier Performance Measures } \\
\hline & & & Accuracy & Sensitivity & Specificity & AUC & $\mathrm{BCR}$ & F- Measure \\
\hline \multirow[t]{9}{*}{ Relief } & \multicolumn{2}{|c|}{ Naïve Bayes } & 71.87 & 0.8636 & 0.400 & 0.6400 & 0.6318 & 0.8085 \\
\hline & \multicolumn{2}{|c|}{ KNN } & 81.25 & 1 & 0.400 & 0.5215 & 0.7000 & 0.8800 \\
\hline & \multicolumn{2}{|l|}{$\mathrm{DA}$} & 71.87 & 0.9090 & 0.300 & 0.2976 & 0.6045 & 0.8163 \\
\hline & \multicolumn{2}{|l|}{ ANN } & 81.25 & 0.6666 & 0.8275 & 0.5277 & 0.7471 & 0.4000 \\
\hline & \multirow[t]{5}{*}{ SVM } & Linear & 81.25 & 0.86 & 0.70 & 0.40 & 0.78 & 0.86 \\
\hline & & RBF & 87.50 & 1 & 0 & 0.43 & 0.50 & 0.93 \\
\hline & & Quadratic & 65.62 & 0.66 & 0.62 & 0.65 & 0.64 & 0.74 \\
\hline & & Polynomial & 68.75 & 0.70 & 0.60 & 0.60 & 0.65 & 0.79 \\
\hline & & MLP & 84.37 & 0.95 & 0.60 & 0.71 & 0.77 & 0.89 \\
\hline \multirow[t]{2}{*}{$\mathrm{mRmR}$} & \multicolumn{2}{|c|}{ Naïve Bayes } & 76.92 & 0.9615 & 0 & 0.7016 & 0.4807 & 0.8695 \\
\hline & \multicolumn{2}{|l|}{ KNN } & 69.69 & 0.76190 & 0.5833 & 0.5873 & 0.6726 & 0.7619 \\
\hline
\end{tabular}




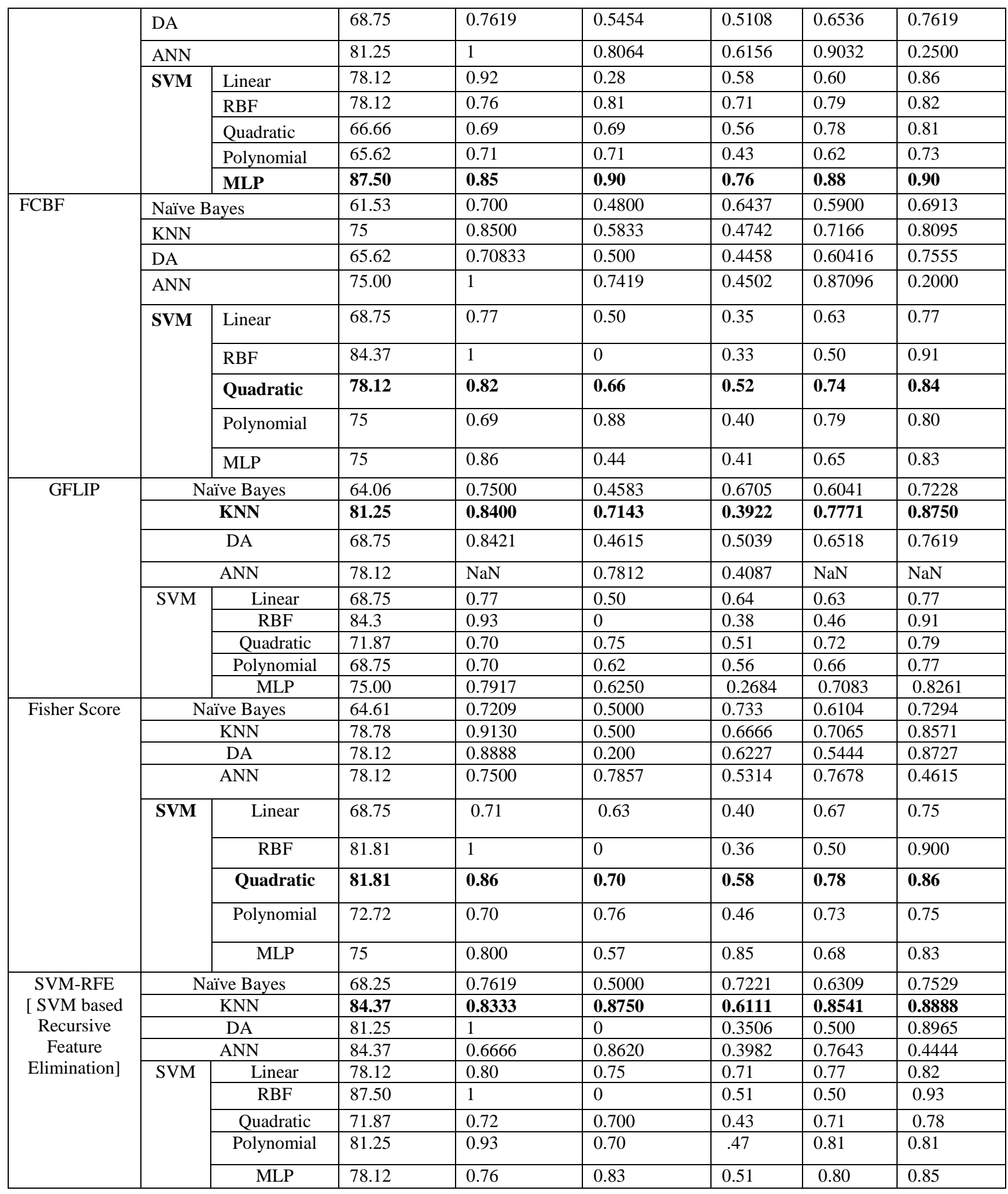

Table 6. Comparison of filter based unsupervised feature selection methods in terms of various classifier performance measures for the given mammogram data set

\begin{tabular}{|l|l|l|l|l|l|l|l|}
\hline \multirow{2}{*}{$\begin{array}{l}\text { Feature } \\
\text { Selection } \\
\text { Methods }\end{array}$} & Classifier for detection & \multicolumn{4}{|l|}{ Classifier Performance Measures } \\
\cline { 3 - 8 } & & Accuracy & Sensitivity & Specificity & AUC & BCR & $\begin{array}{l}\text { F- } \\
\text { Measure }\end{array}$ \\
\hline \multirow{2}{*}{ t-test } & Naïve Bayes & 68.75 & 0.7560 & 0.5652 & 0.6405 & 0.6606 & 0.7560 \\
\cline { 2 - 8 } & KNN & 81.25 & 0.80000 & 0.8571 & 0.5333 & 0.8285 & 0.8695 \\
\hline
\end{tabular}




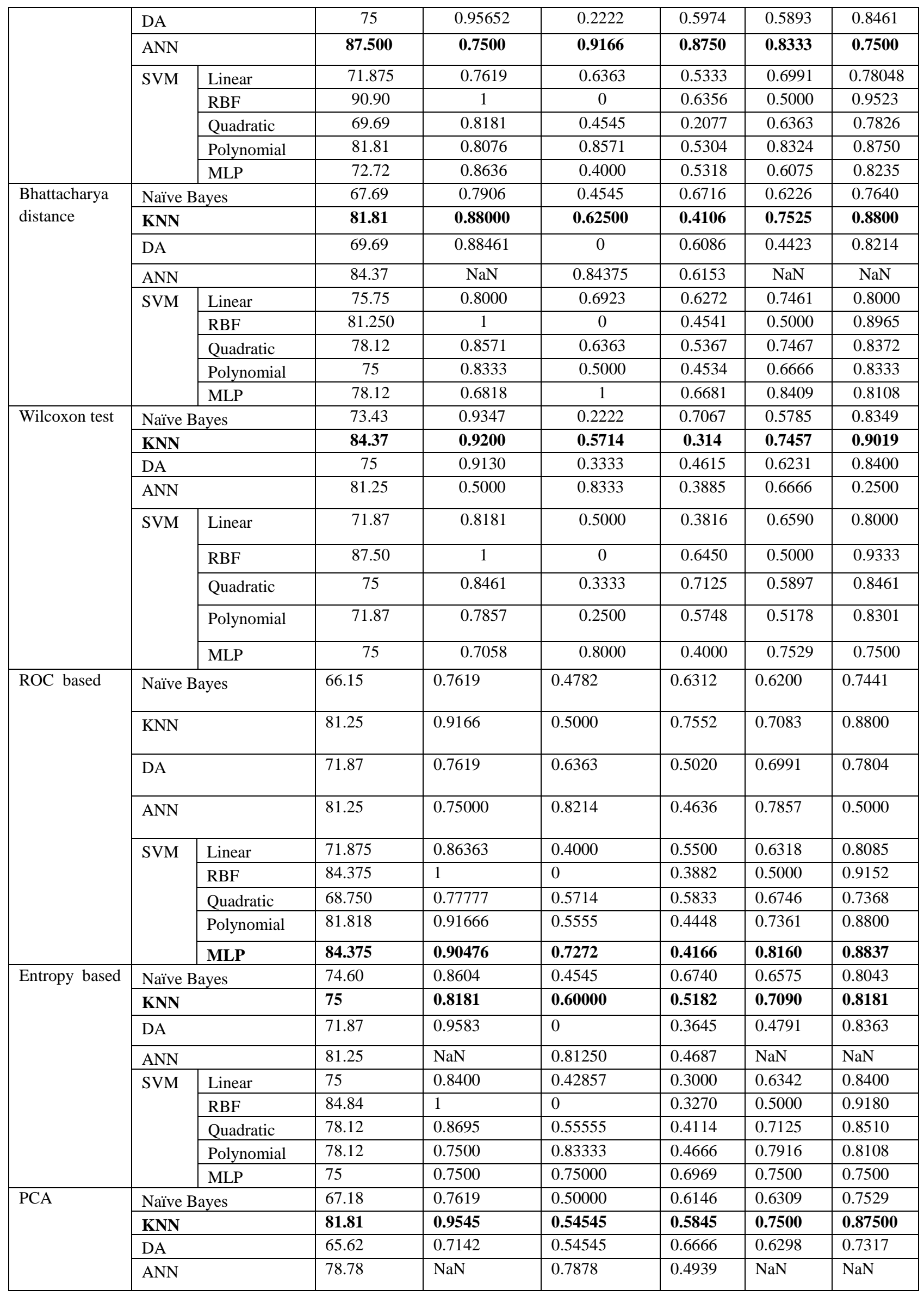




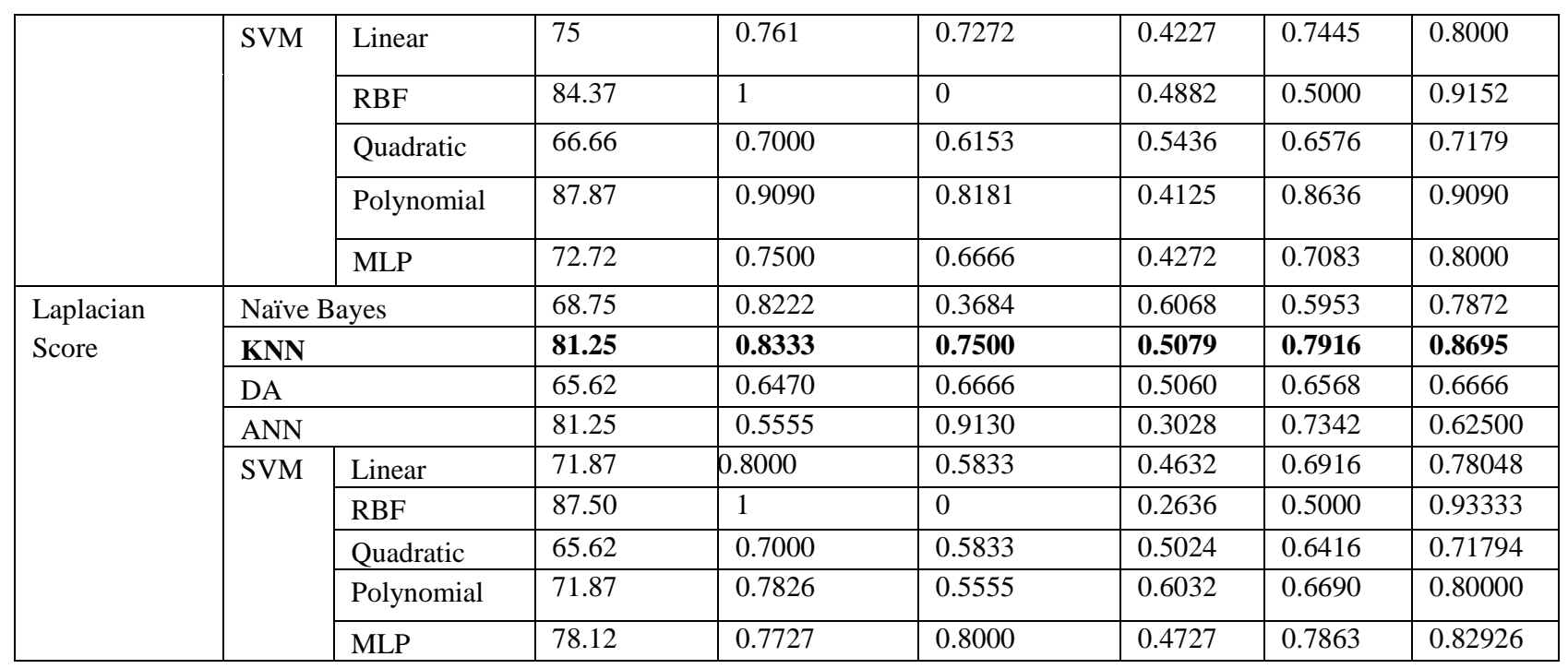
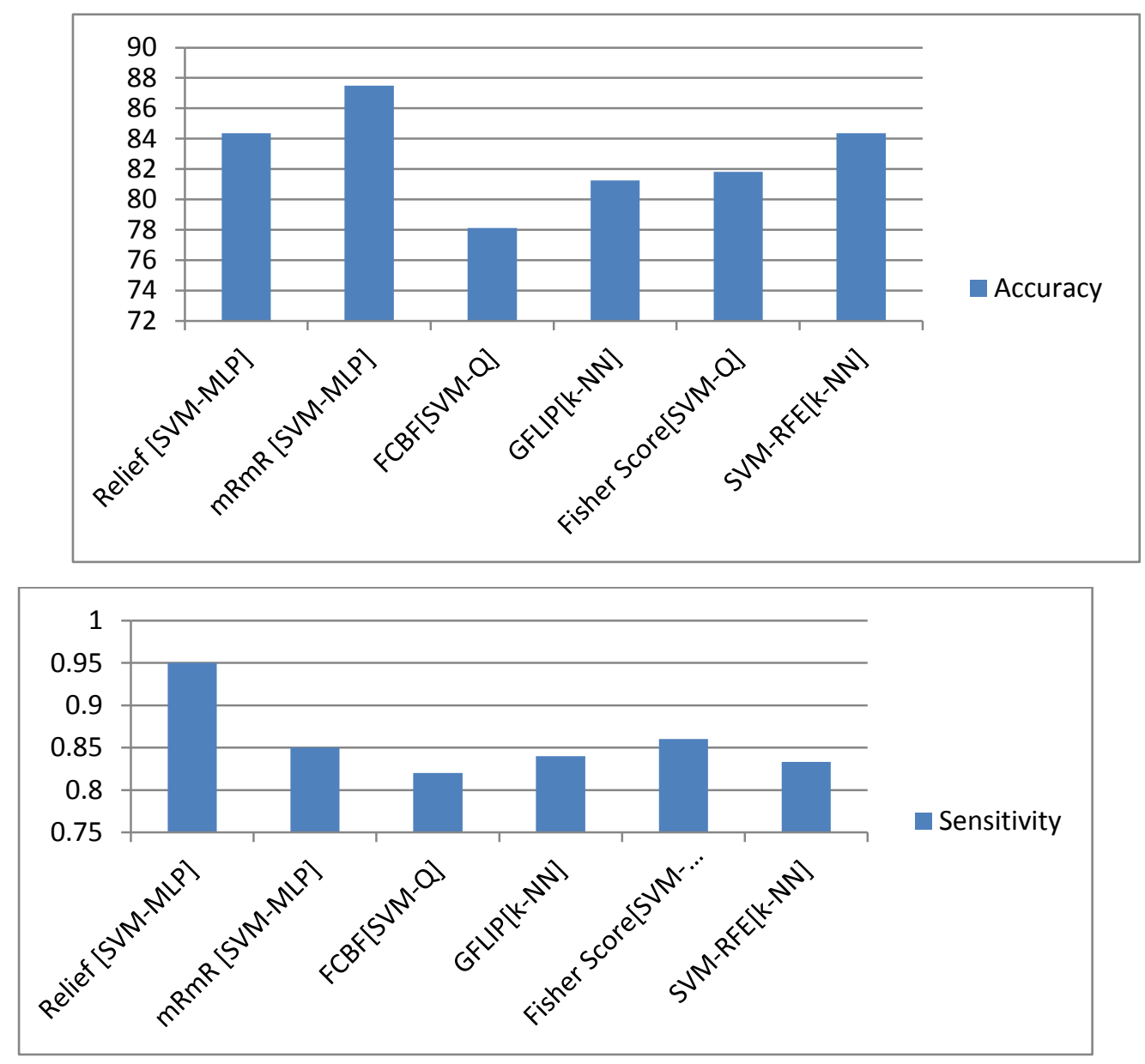


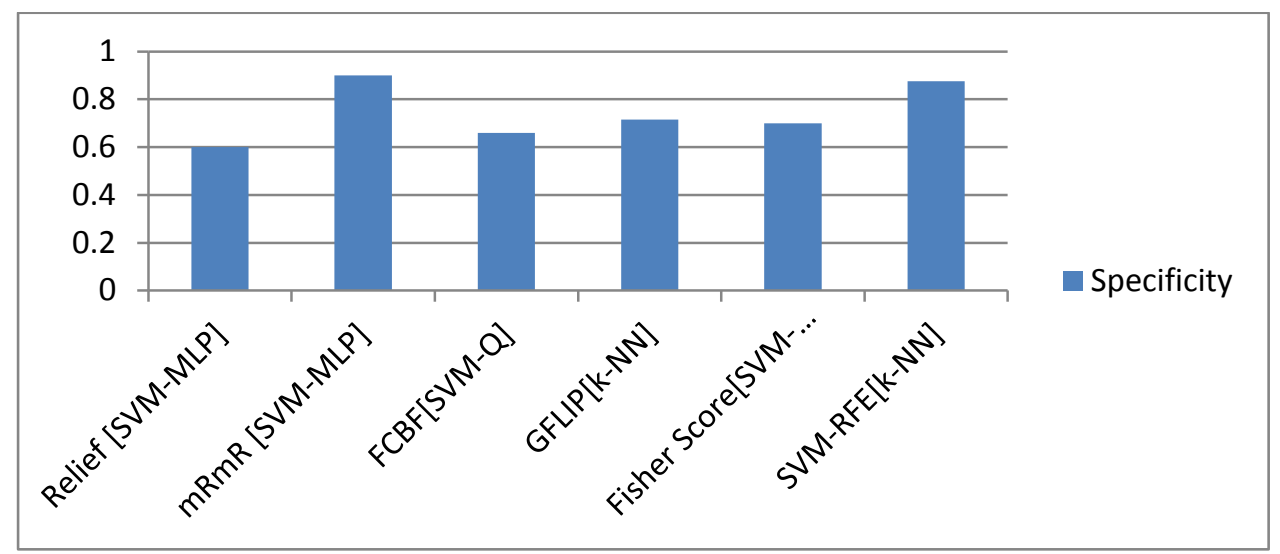

Fig 1: Comparison of supervised filter based feature selection methods in terms of classifier's maximum accuracy , sensitivity, and specificity values
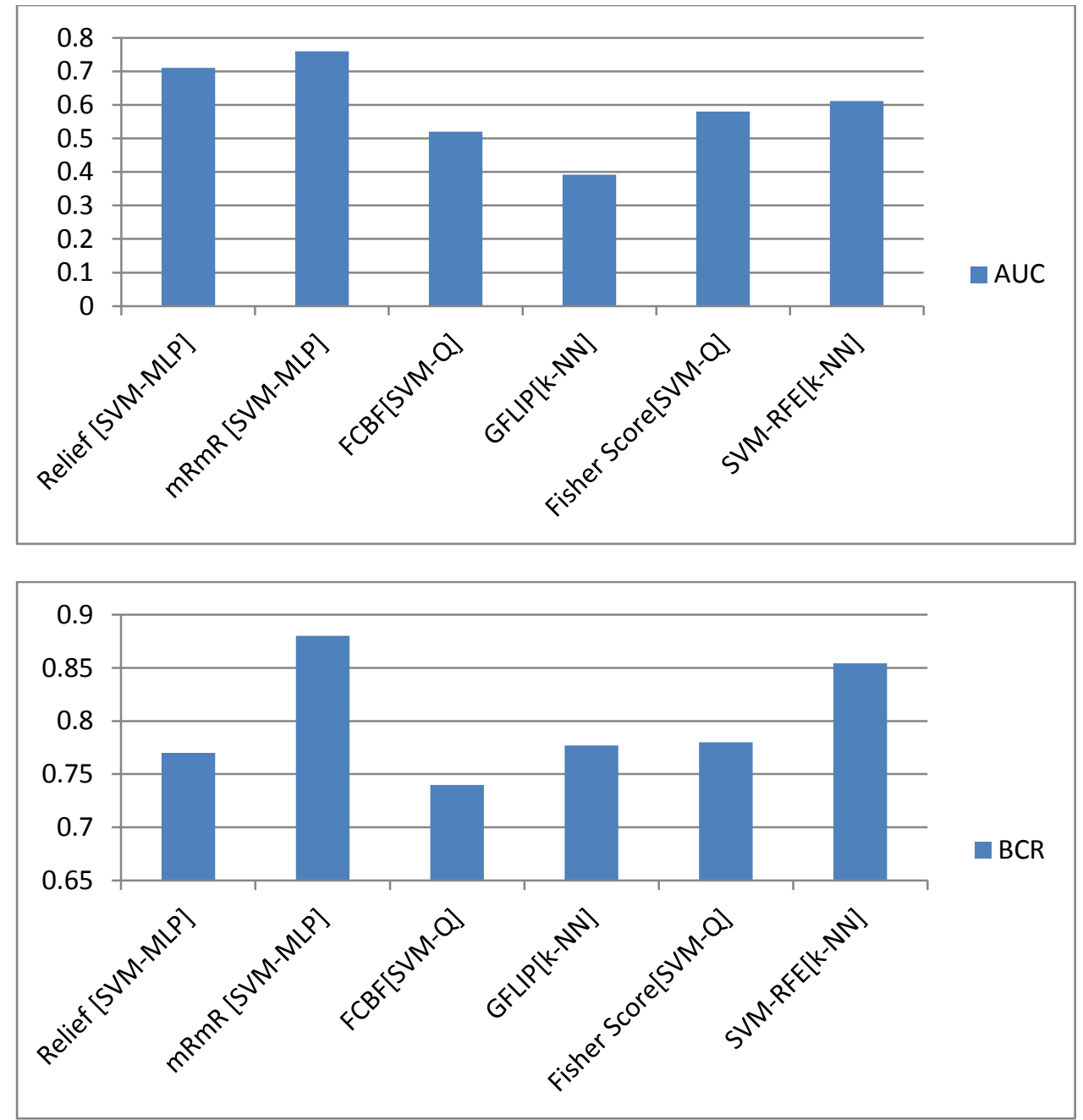


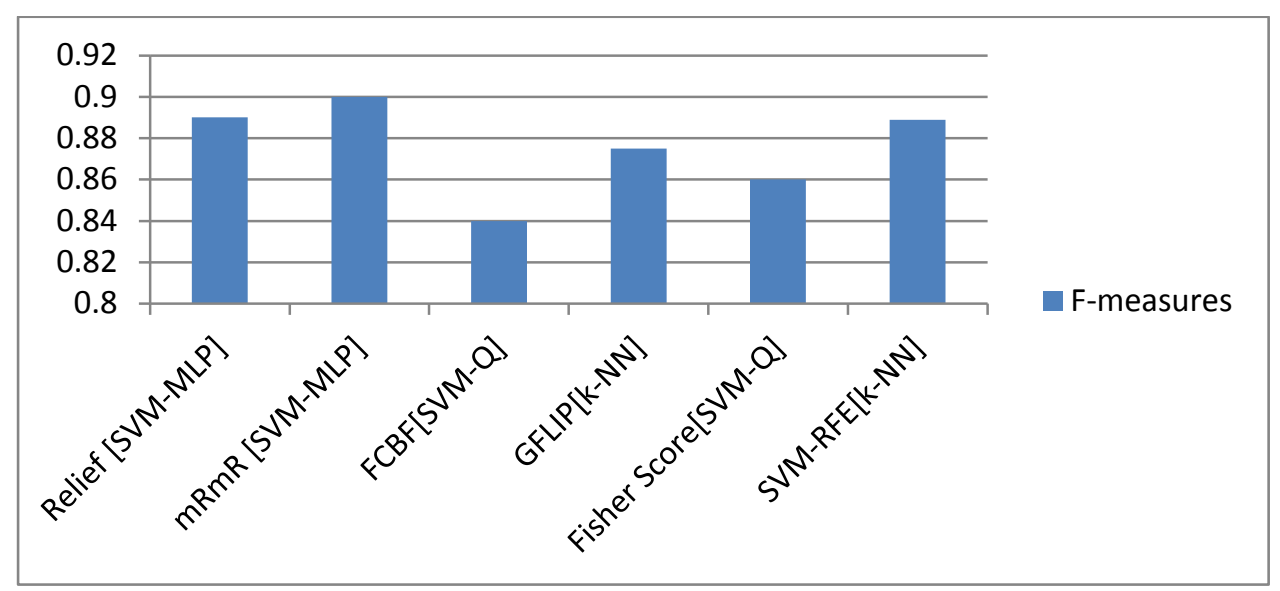

Fig 2: Comparison of supervised filter based feature selection methods in terms of classifier's maximum AUC, BCR, and Fmeasures values
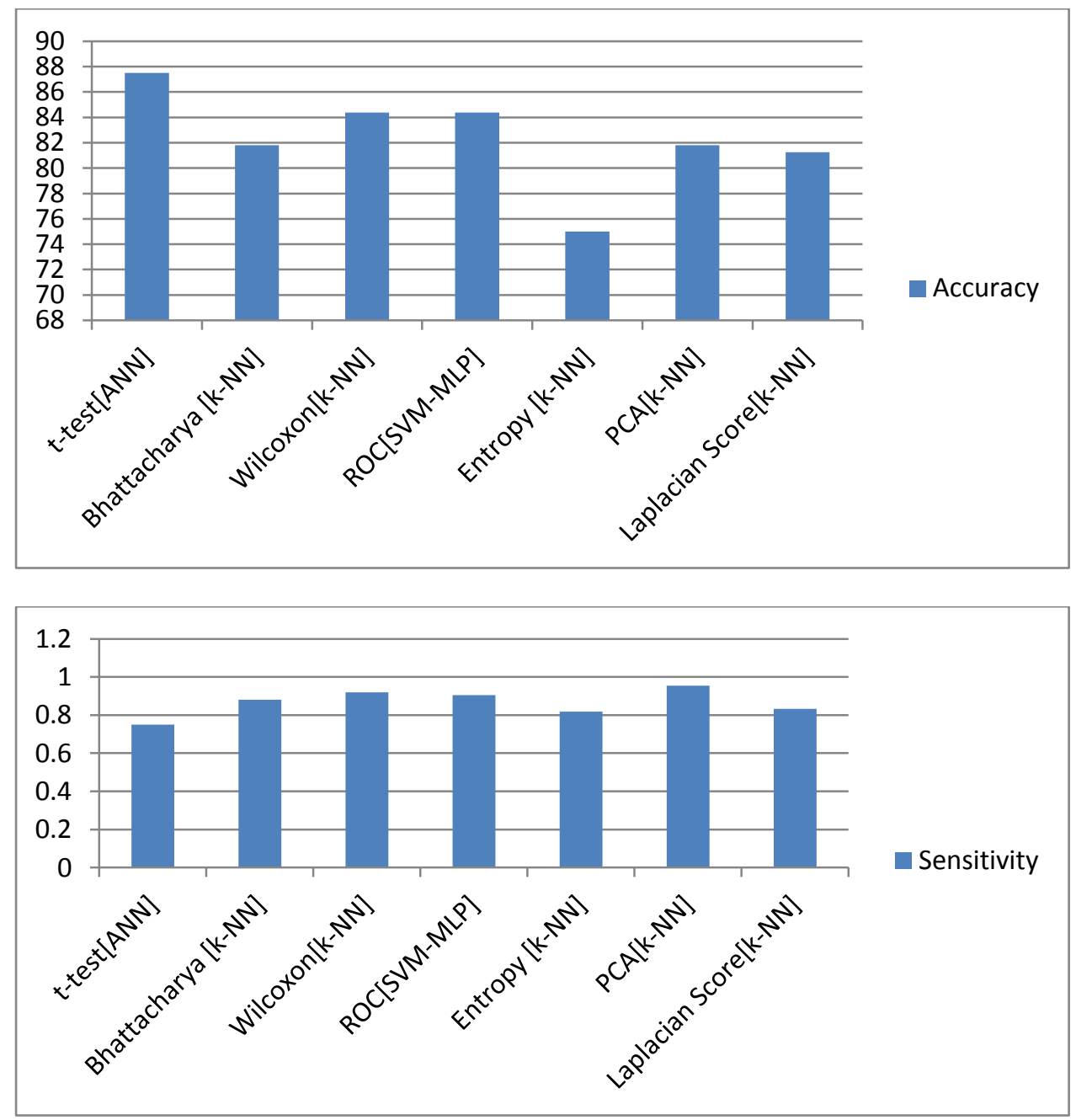


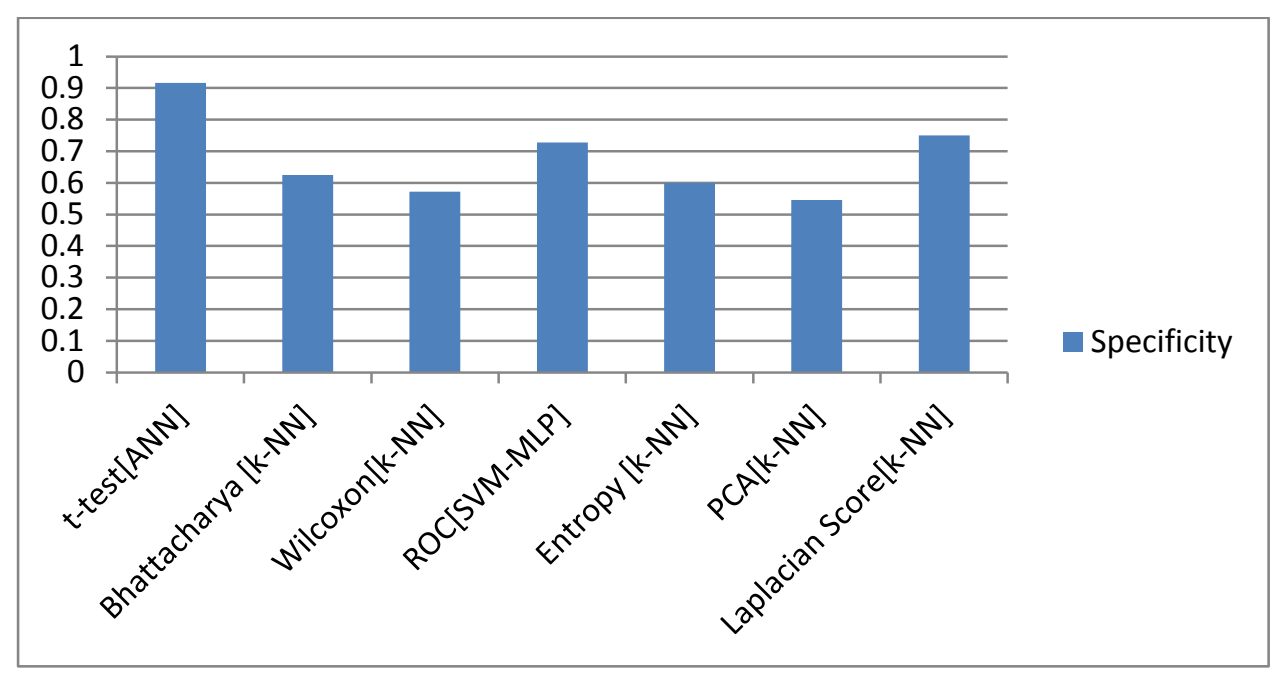

Fig 3: Comparison of unsupervised filter based feature selection methods in terms of classifier's maximum accuracy, sensitivity, and specificity values
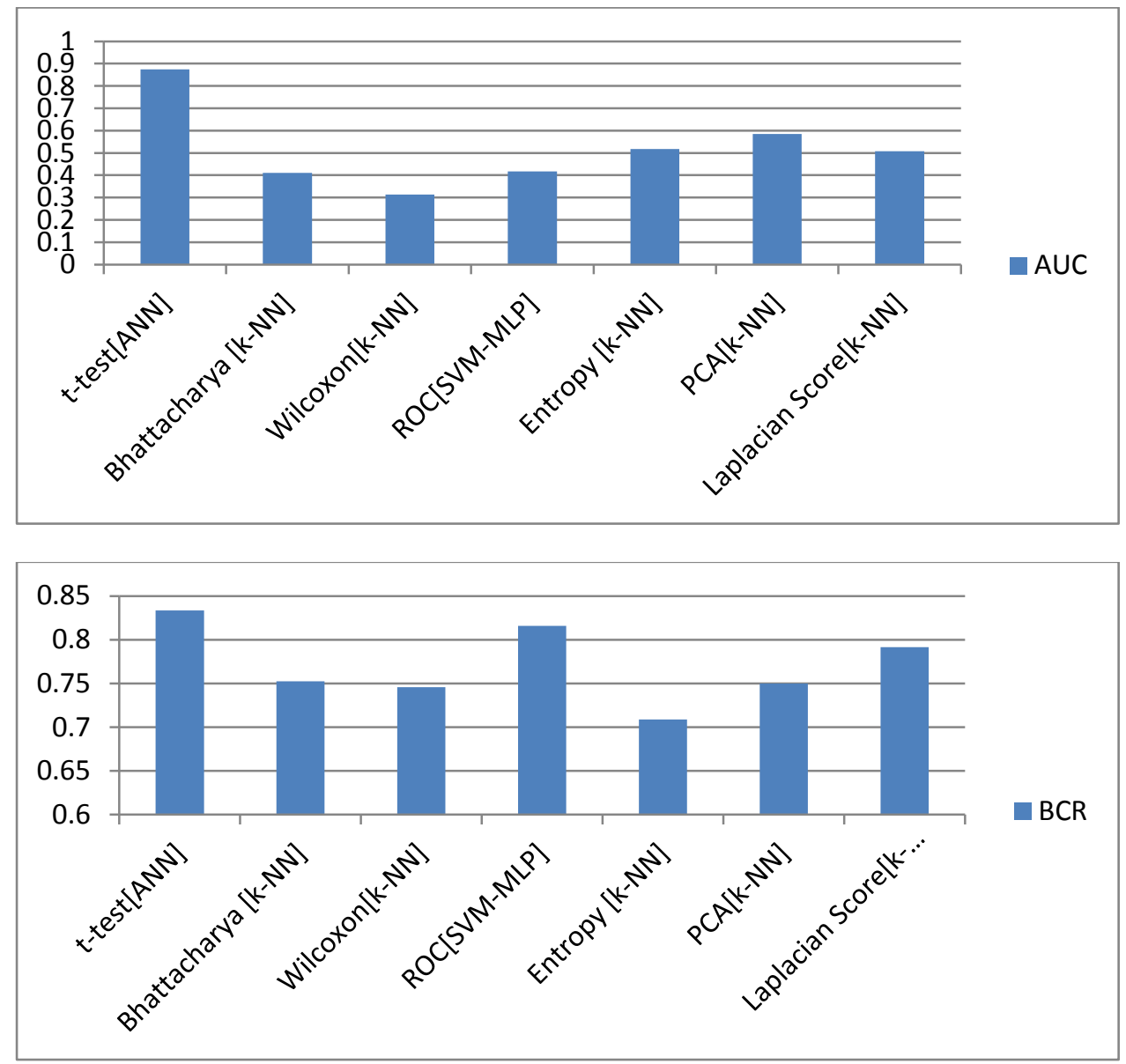


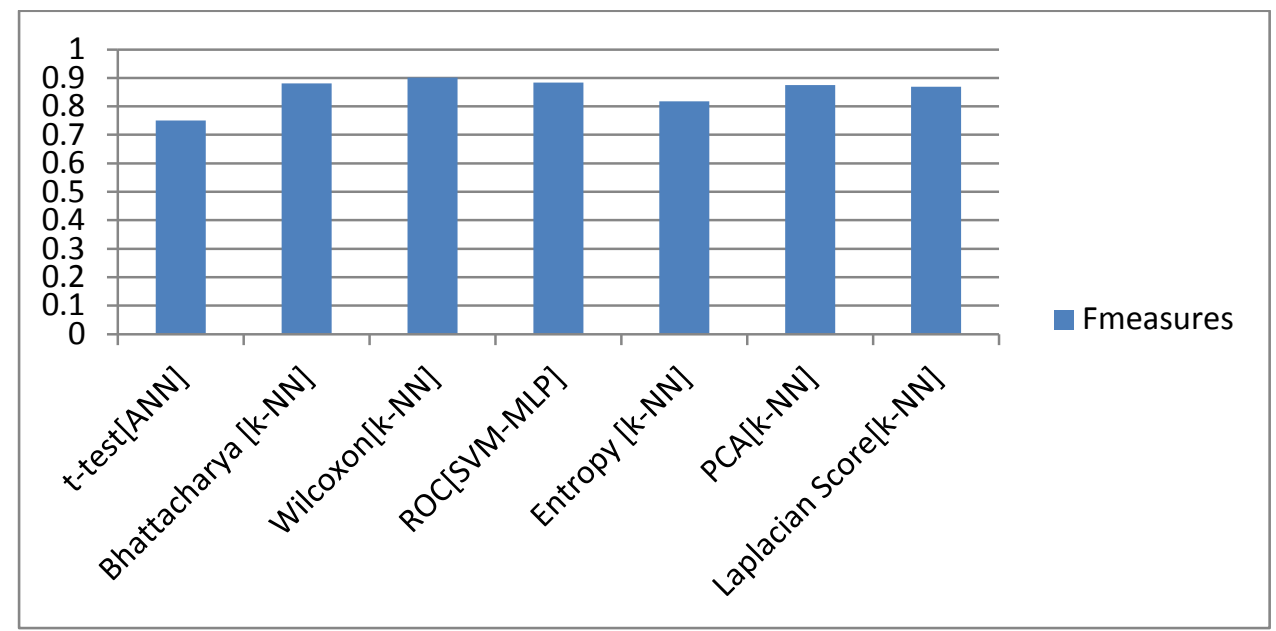

Fig 4: Comparison of unsupervised filter based feature selection methods in terms of classifier's maximum AUC, BCR, and Fmeasures values 